\title{
INDUCTIVE ROTATION TILINGS
}

\author{
DIRK FRETTLÖH AND KURT HOFSTETTER
}

\begin{abstract}
A new method for constructing aperiodic tilings is presented. The method is illustrated by constructing a particular tiling and its hull. The properties of this tiling and the hull are studied. In particular it is shown that these tilings have a substitution rule, that they are nonperiodic, aperiodic, limitperiodic and pure point diffractive.
\end{abstract}

Dedicated to Nikolai Petrovich Dolbilin on the occasion of his 70th birthday.

\section{INTRODUCTION}

The discovery of the celebrated Penrose tilings [12], see also [6, 1], and of physical quasicrystals [15] gave rise to the development of a mathematical theory of aperiodic order. Objects of study are nonperiodic structures (i.e. not fixed by any nontrivial translation) that nevertheless possess a high degree of local and global order. In many cases the structures under consideration are either discrete point sets (Delone sets, see for instance [2]) or tilings (tilings are also called tesselations, see [6] for a wealth of results about tilings). [

Three frequently used construction methods for nonperiodic tilings are local matching rules, cut-and-project schemes and tile substitutions, see [1] and references therein for all three methods. In this paper we describe an additional way to construct nonperiodic tilings, the "inductive rotation", found in 2008 by the second author who is not a scientist but an artist. Variants of this construction have been considered before, see for instance [3] $\rightarrow$ "People" $\rightarrow$ "Petra Gummelt", but up to the knowledge of the authors this construction does not appear in the existing literature.

After describing the construction we will prove that the resulting tilings are nonperiodic, aperiodic and limitperiodic, that they can be described as model sets, hence are pure point diffractive, that they possess uniform patch frequencies and that they are substitution tilings. (For an explanation of these terms see below). The latter property will be the key for most of the other results. We end with some remarks and open questions. In order to keep the paper as much self-contained as possible we try to explain all terms here, but only as far as required to state the results. Where needed we provide references for further information.

Let us fix some notation. A tiling of $\mathbb{R}^{2}$ is a collection of tiles $\left\{Q_{i} \mid i \in \mathbb{N}\right\}$ that is a covering (i.e. $\bigcup_{i \in \mathbb{N}} Q_{i}=\mathbb{R}^{2}$ ) as well as a packing (i.e. the intersection of the interiors of two distinct

UDC Classification 514 

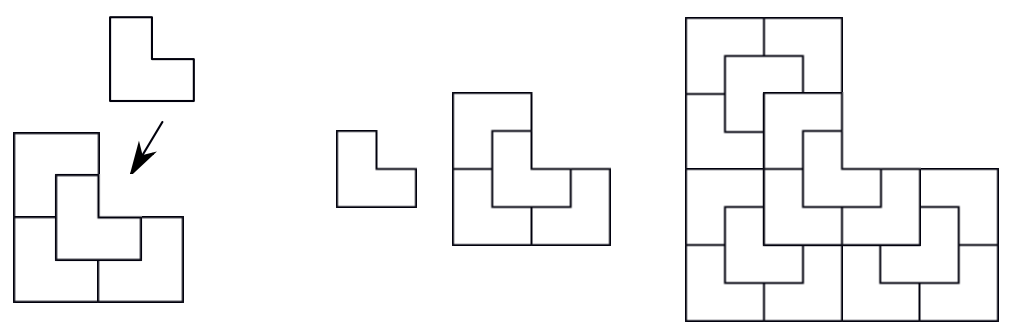

FiguRE 1. The substitution rule for the aperiodic chair tiling (left) and the first two iterates of this rule applied to a single prototile.

tiles $Q_{i}$ and $Q_{j}$ is empty). The $Q_{i}$ are called tiles of the tiling. For our purposes it is fine to think of the tiles as nice compact sets like squares or triangles. If all tiles in the tiling belong to finitely many congruence classes $\left[T_{1}\right], \ldots,\left[T_{m}\right]$ then we call the representatives $T_{1}, \ldots, T_{m}$ prototiles of the tiling. Any finite subset of a tiling is called a patch. Examples of patches of a tiling are obtained by intersecting a tiling $\mathcal{T}$ with a ball $B_{r}(x)$, i.e. with an open ball of radius $r$ with centre $x$. The intersection is defined as

$$
\mathcal{T} \cap B_{r}(x):=\left\{T \in \mathcal{T} \mid T \subset B_{r}(x)\right\} .
$$

Sometimes we want to equip the tiles with an additional attribute like colour or decoration. Then we consider the prototiles equipped with the different colours or decorations, too. One can formalise this by considering pairs $(T, i)$, where $T$ is some tile and $i$ encodes the additional attribute. In order to keep notation simple we keep in mind to distinguish a black unit square from a red unit square when necessary without writing down the additional label $i$.

A vector $t \in \mathbb{R}^{2}$ such that $\mathcal{T}+t=\mathcal{T}$ is called period of $\mathcal{T}$. (If $\mathcal{T}=\left\{Q_{i} \mid i \in \mathbb{N}\right\}$ then $\mathcal{T}+t$ means $\left\{Q_{i}+t \mid i \in \mathbb{N}\right\}$.) A tiling $\mathcal{T}$ is called periodic if it has a nontrivial period, i.e. a period $t \neq 0$. A tiling $\mathcal{T}$ is called 2-periodic if $\mathcal{T}$ possesses two linear independent periods. A tiling $\mathcal{T}$ is called nonperiodic if its only period is the trivial period $t=0$.

A substitution rule $\sigma$ is a simple method to generate nonperiodic tilings. The basic idea is to substitute each prototile $T_{i}$ with a patch $\sigma\left(T_{i}\right)$ consisting of congruent copies of some of the prototiles $T_{1}, \ldots, T_{m}$. The Penrose tilings can be generated by a substitution rule with two prototiles, see [1], [3] or [17]. A simpler example using just one prototiles is shown in Figure 1: a substitution rule for the chair tiling. The chair tiling is nonperiodic, even though it contains large 2-periodic subsets [1].

Given a substitution $\sigma$ with prototiles $T_{1}, \ldots, T_{m}$ a patch of the form $\sigma\left(T_{i}\right)$ is called supertile. More generally, a patch of the form $\sigma^{k}\left(T_{i}\right)$ is called level $k$ supertile. A substitution rule is called primitive if there is $k \in \mathbb{N}$ such that each level $k$ supertile contains congruent copies of all prototiles.

In the sequel we present a construction method for nonperiodic tilings that is similar but not equal to a substitution rule. Before we give a more precise description let us first illustrate the idea of the construction. We start with a square $G_{0}$ of edge length two 

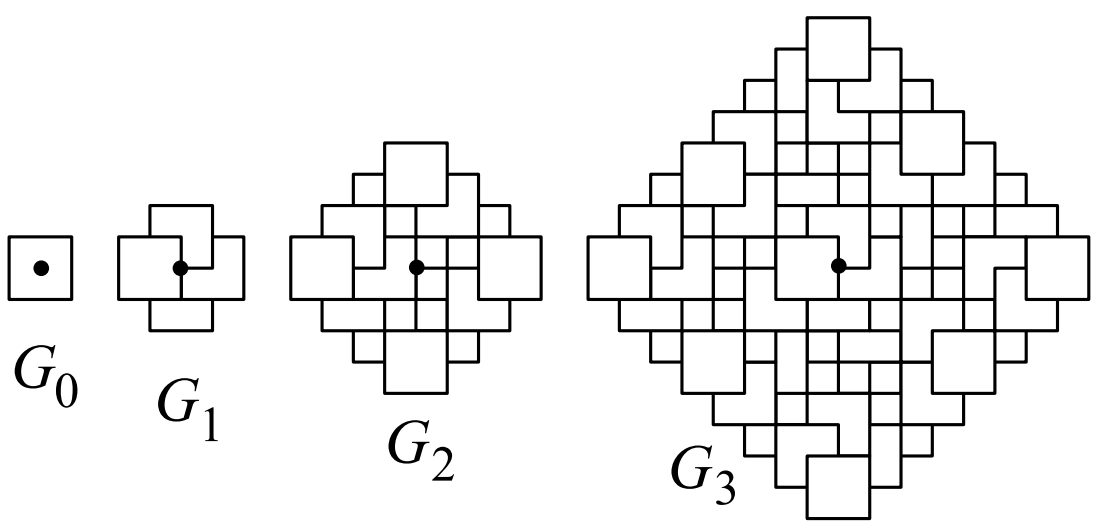

FiguRE 2. The first three iterates of the construction for non-decorated tiles. The black dot indicates the origin.

centred in the origin, see Figure 2 left. (We may imagine this and further squares cut out of cardboard or similar.) In the next step we remove $G_{0}$ (but keep its position in mind); we take four translates of $G_{0}$, and we place the first one one unit to the left with respect to $G_{0}$. The next square we rotate by $\frac{-\pi}{2}$ and place it one unit up with respect to $G_{0}$, shuffling it partly below the first square. The third square we rotate by $-\pi$, place it one unit to the right with respect to $G_{0}$ shuffling it partly below the second square. The last square is rotated by $\frac{-3 \pi}{2}$, placed one unit down with respect to $G_{0}$ and shuffled below the third square. The result is shown in Figure 2 second from the left. Let us call this constellation of four overlapping squares $G_{1}$.

We proceed in similar way: We take four translates of $G_{1}$ and put one translate two units to the left with respect to $G_{1}$, a second translate (rotated by $\frac{-\pi}{2}$ ) two units up with respect to $G_{1}$ and below the first translate, a third translate (rotated by $-\pi$ ) two units to the right with respect to $G_{1}$ and below the second translate, and a fourth translate (rotated by $\frac{-3 \pi}{2}$ ) two units down with respect to $G_{1}$ and below the third translate. The result $G_{2}$, as seen from above, is shown in Figure 2 second from the right.

The next generations are constructed analogously, just replace $G_{1}$ by $G_{n}$ and 2 by $2^{n}$ in the last paragraph. In this way we can cover arbitrary large parts of the plane. In order to translate this covering into a tiling we consider only the visible (parts of) squares. Thus the tiling has four prototiles: a large square with edge length two, a small square with edge length one, a $1 \times 2$ rectangle, and a "chair", i.e. a non-convex hexagon that is the union of three small squares. The more subtle question how this sequence of finite patterns yields an infinite tiling of the plane is answered below. Briefly, we use the fact that the central patches of the $G_{i}$ are fixed under the iteration. These patches yield a nested sequence $S_{2} \subset S_{3} \subset S_{4} \cdots$ of patches with a well defined limit which is an infinite tiling. 


\section{The TILings}

Now we give a more precise description of the construction of the sequence of patches. Let $\varphi$ denote a rotation through $-\pi / 2$ about the origin. Start with a square $Q:=[-1,1]^{2}$. In the following we need to keep track about which squares lie "above" (parts of) other squares. In order to achieve this we may equip each square with an additional label. One may write $(Q, i)$ and consider the label $i$ as the "height" of $Q$ in some orthogonal direction. Anyway, this idea leads to a tedious description of the construction that we omit here. For our purposes it is sufficient just to keep track which squares are higher respectively lower than other squares.

Let $P_{0}:=\{Q\}$. Let

$$
P_{1}:=\left\{Q+(-1,0), \varphi Q+(0,1), \varphi^{2} Q+(1,0), \varphi^{3} Q+(0,-1)\right\},
$$

where the first square is on top of the other squares, the second square is below the first one but above the third and the fourth square, and the fourth square is on bottom. In the $n$th step let $P_{n}$ consist of four congruent copies of $P_{n-1}$ :

$$
P_{n}:=\left\{P_{n-1}+\left(-2^{n-1}, 0\right), \varphi P_{n-1}+\left(0,2^{n-1}\right), \varphi^{2} P_{n-1}+\left(2^{n-1}, 0\right), \varphi^{3} P_{n-1}+\left(0,-2^{n-1}\right)\right\} .
$$

All squares in the first set are on top of the squares in the other three sets, the squares in the second set all are below the squares in the first set but on top of all squares in the third set and the fourth set, and the squares in the fourth set are all below the squares in the other three sets. Two squares within one of the sets $P_{n-1}+t$ inherit their above-below relation from the preceding steps in the iteration.

Note that the centres of the squares are contained in the lattice translate

$$
(1,0)+\Lambda:=(1,0)+\langle(1,1),(1,-1)\rangle_{\mathbb{Z}}=\left\{\left(x_{1}, x_{2}\right) \in \mathbb{R}^{2} \mid x_{1}+x_{2} \text { odd }\right\}
$$

Hence, by the construction, the centre of each of the congruent copies of $P_{1}$ (i.e. the single point that is the intersection of the four squares in the copy of $P_{1}$ ) is contained in $(2,0)+2 \Lambda=(2,0)+\langle(2,2),(2,-2)\rangle_{\mathbb{Z}}$. Since each $x \in(1,1)+\Lambda$ has a unique presentation of the form

$$
x=y+\left\{\begin{array}{l}
(0, \pm 1) \\
( \pm 1,0)
\end{array},(y \in(2,0)+2 \Lambda)\right.
$$

the following lemma is immediate.

Lemma 2.1. The set $P_{n}$ consists of $4^{n}$ congruent copies of $Q$ with centres

$$
\left\{\left(x_{1}, x_{2}\right) \in \mathbb{Z}^{2} \mid x_{1}+x_{2} \text { odd },\left|x_{1}\right|+\left|x_{2}\right| \leq 2^{n}-1\right\}
$$

Because of Lemma 2.1 almost any $\left(x_{1}, x_{2}\right) \in \mathbb{R}^{2}$ (i.e. any with $x_{1}, x_{2} \notin \mathbb{Z}$ ) is covered by exactly two squares in $P_{n}$ (for $n$ large enough). Any $\left(x_{1}, x_{2}\right)$ with exactly one integer coordinate is covered by three squares in $P_{n}$ (the interior of one square and some common edge of two further squares; again if $n$ is large enough), and any $\left(x_{1}, x_{2}\right)$ with two integer coordinates is covered by four squares (midpoints of edges) if $x_{1}+x_{2}$ is even, and by five 


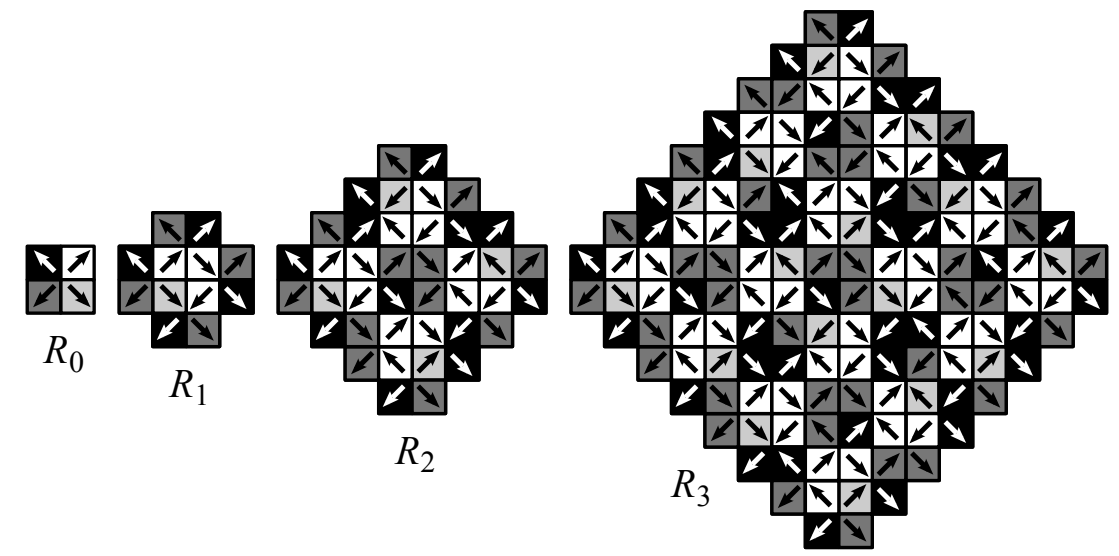

FiguRE 3. The construction of Figure 2 applied to squares with certain decorations. A single square $R_{0}$ is shown on the left: the decoration divides $R_{0}$ into four unit squares of distinct colours, where each unit square carries an arrow in addition. These decorated unit squares yield the tiles of the tiling $\mathcal{A}$.

squares (the centre of one square and the common vertex of four further squares) if $x_{1}+x_{2}$ is odd. This yields the following result.

Lemma 2.2. For all $n \in \mathbb{N}$ the covering degree of $P_{n}$ is two in $\left\{\left(x_{1}, x_{2}\right) \in \mathbb{R}^{2}|| x_{1}+x_{2} \mid \leq\right.$ $\left.2^{n}-1\right\}$.

Since we may decide to equip the squares used above with some additional decoration there are several ways in which this construction yields tilings of $\mathbb{R}^{2}$. In the sequel we will mainly consider one particular tiling: the arrowed tiling $\mathcal{A}$ that is obtained by decorating the underlying square $R_{0}$ with arrows and colours as shown in Figure 3 (left).

The tiling $\mathcal{A}$ is obtained by considering what we see viewing $P_{n}$ from "above", where $P_{n}$ now consists of congruent copies of the decorated square $R_{0}$. Formally, each unit square $K=[k, k+1] \times[m, m+1](k, m \in \mathbb{Z})$ inherits the decoration (colour and arrow) from the top square of the two large squares in $P_{n}$ that contain $K$. We want to consider $\mathcal{A}$ as a tiling by unit squares with decoration as prototiles where the unit squares carry an arrow as well as a colour (here: black, dark grey, light grey, white). From now on the term "tile" always denotes such a unit square with decoration, if not mentioned otherwise.

Regarding the point how to obtain a tiling of the entire plane from the finite patches $P_{n}$, a very natural approach — and a very useful one - is considering the limit $\lim _{n \rightarrow \infty} P_{n}$, where the limit is taken with respect to the local topology [1]. In particular, in this topology two tilings are $\varepsilon$-close if they agree in a ball of radius $1 / \varepsilon$ centred in the origin. For our purposes we may define the distance $d\left(\mathcal{T}, \mathcal{T}^{\prime}\right)$ between two tilings $\mathcal{T}, \mathcal{T}^{\prime}$ by

$$
\widetilde{d}\left(\mathcal{T}, \mathcal{T}^{\prime}\right)=\inf \left\{\varepsilon>0 \mid \exists x, y \in B_{\varepsilon}: B_{1 / \varepsilon} \cap(\mathcal{T}+x)=B_{1 / \varepsilon} \cap\left(\mathcal{T}^{\prime}+y\right)\right\}
$$


where $B_{r}$ denotes the open ball of radius $r$ centred in 0 . In order to make $\widetilde{d}$ into a metric, i.e. in order to ensure transitivity, we define

$$
d\left(\mathcal{T}, \mathcal{T}^{\prime}\right)=\min \left\{\tilde{d}\left(\mathcal{T}, \mathcal{T}^{\prime}\right), \frac{1}{\sqrt{2}}\right\}
$$

compare [9]. We will see in the sequel that the central parts $S_{n}$ of $P_{n}$ for $n \geq 2$ form a nested sequence

$$
S_{2} \subset S_{3} \subset \cdots \subset S_{n} \subset \cdots,
$$

where $S_{n}$ has support $\left\{\left(x_{1}, x_{2}\right)|| x_{1}+x_{2} \mid<2^{n-1}-1\right\}$, hence the sequence $P_{n}$-or any sequence of tilings $\left(\mathcal{T}_{n}\right)_{n}$ where $\mathcal{T}_{n}$ contains $P_{n}$ as its central patch-converges with respect to the metric $d$.

For the next steps it will be convenient to consider the arrowed tiling $\mathcal{A}$. In order to avoid confusion let us call the corresponding sequence $R_{n}$, i.e. the sequence $P_{n}$ where each tile is equipped with the arrow decoration of Figure 3. The first observation is that the arrows on tiles at the boundary of $R_{n}$ point outwards. In order to make the term "boundary" precise, let $\operatorname{bd}\left(R_{n}\right)$ be the set of the tiles in $R_{n}$ that have edges on the boundary of $\bigcup_{T \in R_{n}} T$. These are exactly the tiles that have two edges that are not shared with other tiles of $R_{n}$; or equivalently: these are exactly the tiles with a vertex that is not vertex of another tile of $R_{n}$.

Lemma 2.3. The arrows on all tiles at the boundary $\operatorname{bd}\left(R_{n}\right)$ of $R_{n}$ point outwards, i.e. in the direction of their vertex that is not a vertex of any other tile of $R_{n}$.

Proof. The claim is true for $R_{0}$ and $R_{1}$, hence it follows inductively for all $R_{n}$ by considering the construction: The boundary of $R_{n}$ consists of the boundaries of $R_{n-1}$.

Lemma 2.4. All arrows on the tiles in $R_{n}$ on the two diagonals $\left(x_{1}, x_{2}\right)$ with $x_{1}=x_{2}$ or $x_{1}=-x_{2}$ respectively show in the following directions: $(1,-1)$ for $x_{1}=x_{2},(1,1)$ for $x_{1}=-x_{2}<0,(-1,-1)$ for $x_{1}=-x_{2}>0$.

This is an immediate consequence of Lemma 2.3 and the construction. Figure 4 (right) illustrates the situation.

Theorem 2.5. The sequence $R_{n}$ is convergent with respect to the metric $d$. Consequently, any sequence of tilings $\left(\mathcal{T}_{n}\right)_{n}$ where $\mathcal{T}_{n}$ contains $R_{n}$ as its central patch is convergent with respect to the metric $d$.

Proof. The idea is to consider three steps of the iteration, i.e. how $R_{n+2}$ is build up from congruent copies of $R_{n-1}$. This shows that the central patch of $R_{n+1}$ reappears as the central patch of $R_{n+2}$, compare Figure 5 .

For $n \geq 1$ the set $R_{n}^{\prime}:=R_{n} \backslash \operatorname{bd}\left(R_{n}\right)$ is nonempty and reappears in $R_{n+1}$, see Figure 4 . Considering how $R_{n}^{\prime}$ is contained in $R_{n+1}$ we find that the central patch $S$ of $R_{n+1}$ consists of four congruent copies of $R_{n-1}^{\prime}$. (This is indicated in the left part of Figure 5 , the area shaded in darker grey.) The central patch of $R_{n+2}$, indicated in Figure 5 by shading in 

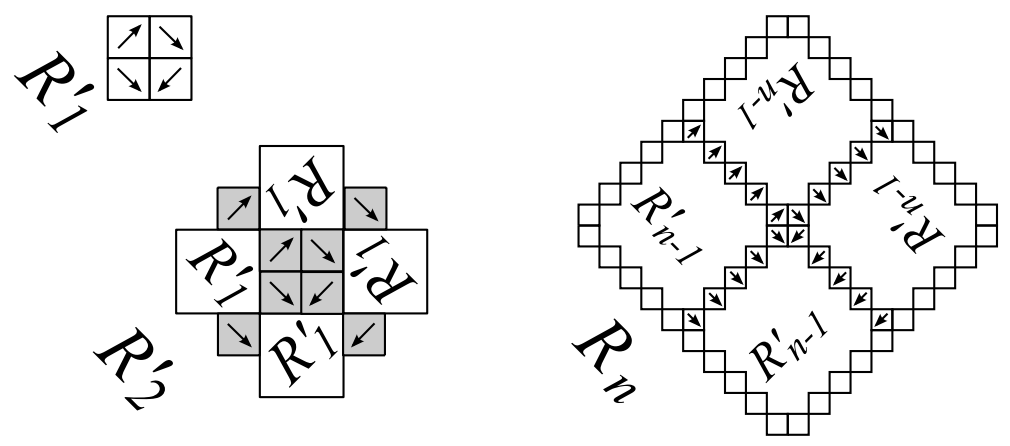

Figure 4. The structure of the patches $R_{n}^{\prime}$. Each $R_{n}^{\prime}$ is build from four congruent copies of $R_{n-1}^{\prime}$ and some further tiles on its main diagonals. The arrows in the tiles on the diagonals are pointing in the directions given in Lemma 2.4.

lighter grey, is a translate of the central patch $S$ of $R_{n+1}^{\prime}$, since (a) it is build up from congruent copies of $R_{n-1}^{\prime}$ in the same manner (this yields the bulky part), and (b) the arrows on the diagonal boundaries of the $R_{n-1}^{\prime}$ coincide by Lemma 2.4 together with the construction (this yields the "skeleton" part). The directions of the arrows on the tiles on the boundaries of the $R_{n-1}^{\prime}$ are indicated by small black squares in the corresponding corners of the tiles in Figure 5 .

Altogether this yields that the central patch of $R_{n+1}$ equals the central patch of $R_{n+2}$. Let us denote the central patch of $R_{n+1}$ consisting of four congruent copies of $R_{n-1}^{\prime}$ (plus the tiles on the two diagonals) by $S_{n+1}$. More precisely, let

$S_{n+1}:=R_{n+1} \cap\left\{\left(x_{1}, x_{2}\right)|| x_{1}+x_{2} \mid \leq 2^{n-1}-1\right\}=R_{n+2} \cap\left\{\left(x_{1}, x_{2}\right)|| x_{1}+x_{2} \mid \leq 2^{n-1}-1\right\}$.

The patches $S_{n+1}$ yield a nested sequence

$$
S_{2} \subset S_{3} \subset S_{3} \subset \cdots \subset S_{n} \subset \cdots .
$$

In particular we obtain that for any $n \in \mathbb{N}$ we have that $R_{n+1}$ coincides with all $R_{k}$ $(k \geq n+1)$ in a ball $B_{1 / \varepsilon}$ for $\frac{1}{\varepsilon} \leq \frac{1}{\sqrt{2}}\left(2^{n-1}-1\right)$, i.e. $\varepsilon \geq \frac{\sqrt{2}}{2^{n-1}-1}$. Thus

$$
d\left(R_{n+1}, R_{k}\right) \leq \frac{\sqrt{2}}{2^{n-1}-1} \quad \text { for } k \geq n+1 .
$$

Hence the sequence $R_{n}$, respectively any sequence of tilings $\left(\mathcal{T}_{n}\right)_{n}$ where $\mathcal{T}_{n}$ contains $R_{n}$ as its central patch, converges for $n \rightarrow \infty$ with respect to the metric $d$.

Note that neither the sets $R_{n}$ nor the sets $R_{n}^{\prime}$ form a nested sequence, there are slight mismatches further away from the centre.

Since the sequence $R_{n}$, respectively any sequence of tilings $\left(\mathcal{T}_{n}\right)_{n}$ where each $\mathcal{T}_{n}$ contains $R_{n}$ as its central patch, converges with respect to $d$, there is a unique $\operatorname{limit} \mathcal{A}=\lim _{n \rightarrow \infty} \mathcal{T}_{n}$. The tiling $\mathcal{A}$ is the desired infinite tiling. Moreover we may now define the hull of $\mathcal{A}$. In 


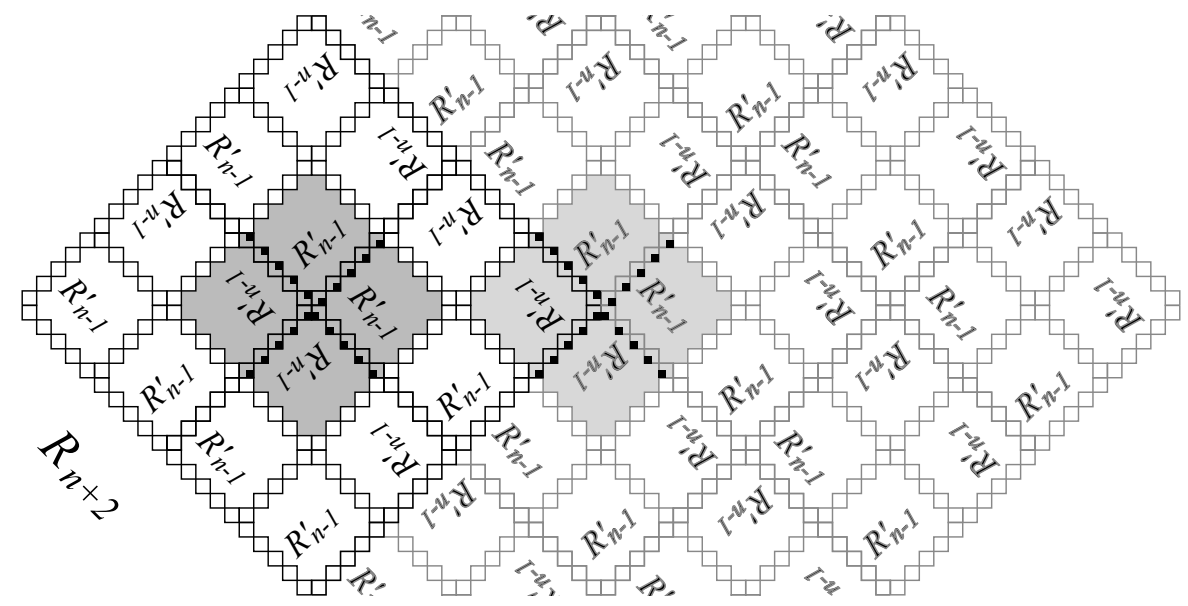

FiguRE 5. How congruent copies of $R_{n-1}^{\prime}$ are located within $R_{n+1}$ (left part) and $R_{n+2}$ (everything).

the theory of aperiodic tilings the hull turns out to be a central object of study. The hull is the closure of the set of all translates of $\mathcal{A}$, i.e.

$$
\mathbb{X}(\mathcal{A})=\overline{\left\{\mathcal{A}+t \mid t \in \mathbb{R}^{2}\right\}}
$$

where $\bar{M}$ defines the closure of the set $M$ with respect to $d$, see again [1, Chapter 4] for details.

Since $\mathcal{A}$ has only finitely many prototiles, and all tiles are vertex-to-vertex, the set of all finite patches in $\mathcal{A}$ up to some given radius $r>0$ is finite, up to congurence (even up to translations). This property is called finite local complexity [1]. It holds for each tiling in $\mathbb{X}(\mathcal{A})$ and for the set $\mathbb{X}(\mathcal{A})$ as a whole. Hence by standard reasoning ([13, 14, see also [1. Chapter 4]), $\mathbb{X}(\mathcal{A})$ is compact with respect to $d$, hence $(\mathbb{X}(\mathcal{A}), d)$ is a compact metric space.

\section{Properties of the tilings}

With the help of the metric $d$ above we are now able to give a precise definition of aperiodic tilings. A tiling is aperiodic if its hull does not contain any periodic tiling. In particular, aperiodicity implies nonperiodicity. (The hull of a periodic tiling $\mathcal{T}$ contains only translates of $\mathcal{T}$, so in particular it contains only periodic tilings.)

Considering Figure 5 one may get the impression that $\mathcal{A}$ is periodic. At least the arrangement of the $R_{n-1}^{\prime}$ in the image seem to form a periodic pattern. This behaviour is well-known for certain aperiodic structures like the chair tiling, the Robinson square tiling or the one-dimensional period doubling sequence, see [1, 3]. Loosely speaking, a limitperiodic tiling is one that is the union of infinitely many periodic packings with larger and larger periods where the lattices $\Lambda_{i}$ of periods are nested sequences $\Lambda_{1} \subset \Lambda_{2} \subset \Lambda_{3} \cdots$, possibly up to a set of density zero. In fact, the exact definition of limitperiodicity uses spectral 


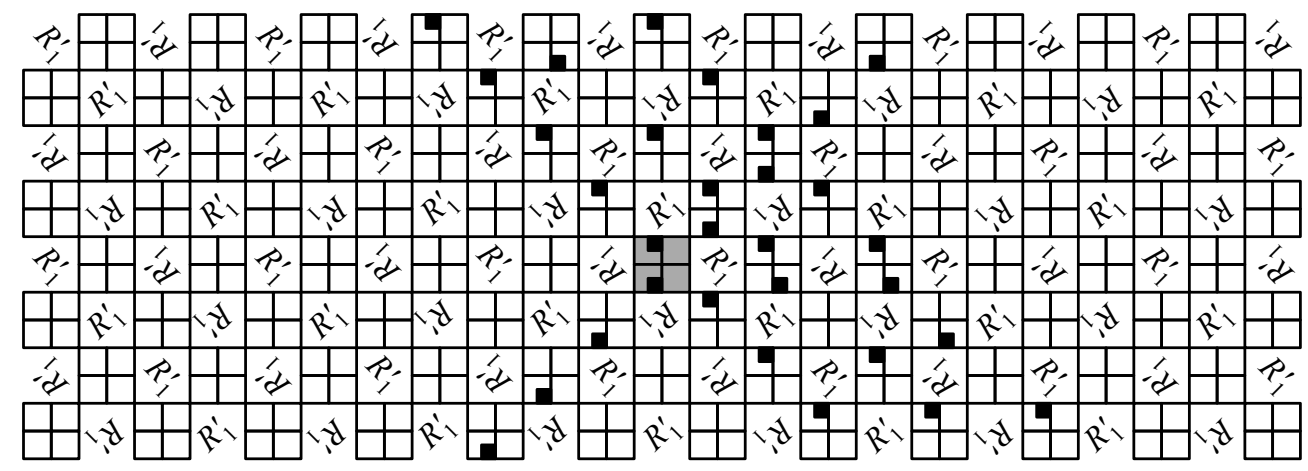

Figure 6 . The congruent copies of $R_{1}^{\prime}$ in $\mathcal{A}$ form a periodic subset of the tiling with density $\frac{1}{2}$. The centre of the shaded patch is the origin.

properties of the hull. We will not explain this in detail (compare [1]) since there is a simple geometric sufficient condition ensuring limitperiodicity that applies to our examples.

Theorem 3.1. $\mathcal{A}$ is the union of 2-periodic packings $M_{n}$ and a set $\mathcal{Z}$ of density zero. More precisely, $\mathcal{A}=\mathcal{Z} \cup \bigcup_{n \in \mathbb{N}} M_{n}$, where

$$
\begin{array}{r}
M_{n}:=\left\{R_{n}^{\prime}+\left(\left(2^{n}, 0\right)+2^{n+1} \Lambda\right), \varphi R_{n}^{\prime}+\left(\left(0,-2^{n}\right)+2^{n+1} \Lambda\right), \varphi^{2} R_{n}^{\prime}+\left(\left(-2^{n}, 0\right)+2^{n+1} \Lambda\right),\right. \\
\left.\varphi^{3} R_{n}^{\prime}+\left(\left(0,2^{n}\right)+2^{n+1} \Lambda\right)\right\}
\end{array}
$$

where $\Lambda=\langle(1,1),(1,-1)\rangle_{\mathbb{Z}}$ (i.e. the integer span of the two vectors $(1,1)$ and $(1,-1)$ ) and $\mathcal{Z}$ is the set of all tiles in $\mathcal{A}$ whose diagonals are contained in $\left\{\left(x_{1}, x_{2}\right) \mid x_{1}= \pm x_{2}\right\}$.

Proof. By the proof of Theorem 2.5 we get that for any $n \in \mathbb{N}$ the central patch of $\mathcal{A}$ is $R_{n}$. Considering how patches of type $R_{n-3}^{\prime}$ are located in $R_{n}$ we obtain inductively arbitrary large parts of 2-periodic packings consisting of congruent copies of $R_{k}^{\prime}, \varphi R_{k}^{\prime}, \varphi^{2} R_{k}^{\prime}$ and $\varphi^{3} R_{k}^{\prime}$ for any $k \in \mathbb{N}$, compare Figure 5 . Figure 6 indicates the arrangement of congruent copies of $R_{1}^{\prime}$. Let $\Lambda=\langle(1,1),(1,-1)\rangle_{\mathbb{Z}}$. Then the four 2-periodic sets

(1) $R_{1}^{\prime}+((2,0)+4 \Lambda), \varphi R_{1}^{\prime}+((0,-2)+4 \Lambda), \varphi^{2} R_{1}^{\prime}+((-2,0)+4 \Lambda), \varphi^{3} R_{1}^{\prime}+((0,2)+4 \Lambda)$

yield already half of the tiles of $\mathcal{A}$. Each of the four sets has period vectors $(4,4)$ and $(4,-4)$. This situation is indicated in Figure 6. The arrangement of $R_{n}$ is similar on all levels $2^{n}$, hence further periodic structures in $\mathcal{A}$ are

$$
\begin{array}{r}
R_{n}^{\prime}+\left(\left(2^{n}, 0\right)+2^{n+1} \Lambda\right), \varphi R_{n}^{\prime}+\left(\left(0,-2^{n}\right)+2^{n+1} \Lambda\right), \varphi^{2} R_{n}^{\prime}+\left(\left(-2^{n}, 0\right)+2^{n+1} \Lambda\right) \\
\varphi^{3} R_{n}^{\prime}+\left(\left(0,2^{n}\right)+2^{n+1} \Lambda\right)
\end{array}
$$

For each $n$ the union of the four sets has density $\frac{1}{2}$. Each of the four sets has period vectors $\left(2^{n+1}, 2^{n+1}\right)$ and $\left(2^{n+1},-2^{n+1}\right)$. It is easy to see that the union of these sets for all $n \in \mathbb{N}$ contains all tiles of $\mathcal{A}$ except tiles along the diagonals $x_{1}= \pm x_{2}$, i.e. all tiles in $\mathcal{Z}$. This set has density zero. 


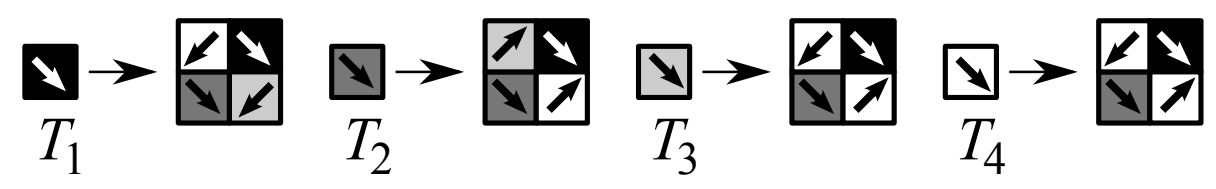

Figure 7 . The substitution rule $\sigma$ yielding the tiling $\mathcal{A}$.

Remark 3.2. Note that Equation (2) yields all tiles in $\mathcal{A}$ except the tiles on the diagonals $x_{1}= \pm x_{2}$, hence (2) together with Lemma 2.4 yields the entire tiling.

The next theorem is the key to all further results. This holds because the theory of substitution tilings is developed pretty well, while there is no theory for the construction above yet.

Theorem 3.3. $\mathcal{A}$ can be generated by a primitive tile substitution rule.

Proof. This result is achieved by giving an appropriate substitution rule yielding the tiling $\mathcal{A}$. More precisely, we will show that this rule yields a substitution tiling that possesses the particular structure (union of periodic packings) described by Remark 3.2 .

This substitution rule is shown in Figure 7. Let us denote this substitution rule by $\sigma$. Its prototiles are denoted by $T_{1}, T_{2}, T_{3}$ and $T_{4}$; they are the prototiles of $\mathcal{A}$. Figure 8 shows the action of $\sigma, \sigma^{2}, \sigma^{3}$ and $\sigma^{4}$ on $T_{1}$. Note that $\sigma\left(T_{3}\right)=\sigma\left(T_{4}\right)$. Note also that there are no reflections involved: all tiles in $\sigma\left(T_{i}\right)$ are direct congruent copies of the $T_{i}$, not reflected congruent copies.

To obtain an infinite substitution tiling in $\mathbb{X}_{\sigma}$ it is useful to consider a tiling $\mathcal{S}$ that is fixed under $\sigma$, i.e. $\sigma(\mathcal{S})=\mathcal{S}$. The construction of such a tiling is standard: take a legal "seed", i.e. a patch $P$ that is contained in some $\sigma^{n}(T)$ for some prototile $T$, such that $\sigma(P)$ (or $\sigma^{2}(P)$ or $\left.\sigma^{3}(P) \ldots\right)$ contains a translate of $P$ in its interior. Here we choose $P$ as the patch of four tiles shown in Figure 9 left, consisting of three dark grey tiles of type $T_{2}$ and one black tile of type $T_{1}$. $P$ occurs in $\sigma^{4}\left(T_{1}\right)$, see Figure 8 .

Applying $\sigma$ to $P$ one gets that $P$ is exactly the central patch of $\sigma(P)$, compare Figure 9 . Hence $\sigma(P)$ is the central patch of $\sigma^{2}(P)$, and inductively we get that $\sigma^{n}(P)$ is the central patch of $\sigma^{n+1}(P)$. Hence the sequence $\sigma_{n}(P)$ (or any sequence of tilings $\mathcal{S}_{n}$ having $\sigma^{n}(P)$ as their central patch respectively) converge to some tiling in the local topology. Let us denote this tiling by $\mathcal{S}$.

In order to show that $\mathcal{S}=\mathcal{A}$ we use the particular structure of $\mathcal{A}$ stated in Remark 3.2. Consider $\sigma^{3}\left(T_{1}\right), \sigma^{3}\left(T_{2}\right), \sigma^{3}\left(T_{3}\right)$ and $\sigma^{3}\left(T_{4}\right)$. The interior of each of the four patches contains four congruent copies of $R_{1}^{\prime}$. On their boundaries these patches have halves of $R_{1}^{\prime}$. (See for instance Figure 8, the patch on the right is the union of one copy of $\sigma^{3}\left(T_{1}\right), \sigma^{3}\left(T_{2}\right), \sigma^{3}\left(T_{3}\right)$ and $\sigma^{3}\left(T_{4}\right)$ each.) The entire constellation of congruent copies of $R_{1}^{\prime}$ and halves of $R_{1}^{\prime}$ agrees on all four supertiles $\sigma^{3}\left(T_{i}\right)$, and it is invariant under rotation by $\pi / 2$ about the centre of each $\sigma^{3}\left(T_{i}\right)$. All tiles in $\mathcal{S}$ lie edge to edge, hence all "supertiles" $\sigma^{3}\left(T_{i}\right)$ lie edge-to-edge. Hence $\mathcal{S}$ contains the same periodic arrangement as $\mathcal{A}$, i.e. the one in Equation (1). 


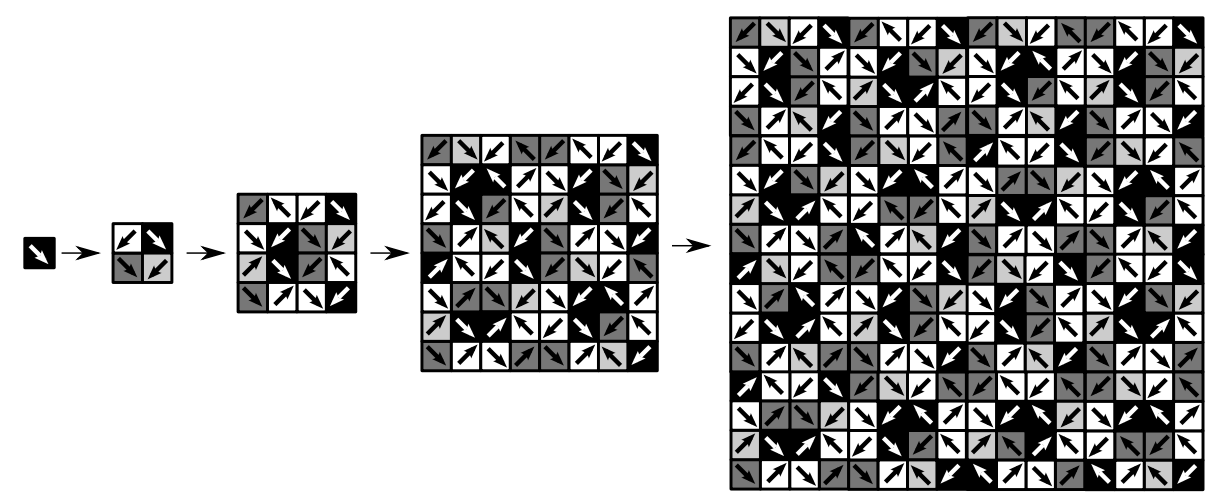

Figure 8. The image shows $T_{1}, \sigma\left(T_{1}\right), \sigma^{2}\left(T_{1}\right), \sigma^{3}\left(T_{1}\right)$, and $\sigma^{4}\left(T_{1}\right)$ (from left to right). On the right it is indicated how $\sigma^{4}\left(T_{1}\right)$ consists of congruent copies of $\sigma^{3}\left(T_{1}\right), \sigma^{3}\left(T_{2}\right), \sigma^{3}\left(T_{3}\right)$ and $\sigma^{3}\left(T_{1}\right)$. A seed for $\mathcal{A}$ is found in $\sigma^{4}\left(T_{1}\right)$ (e.g. the middle of the fourth and the fifth row, compare Figure 9).

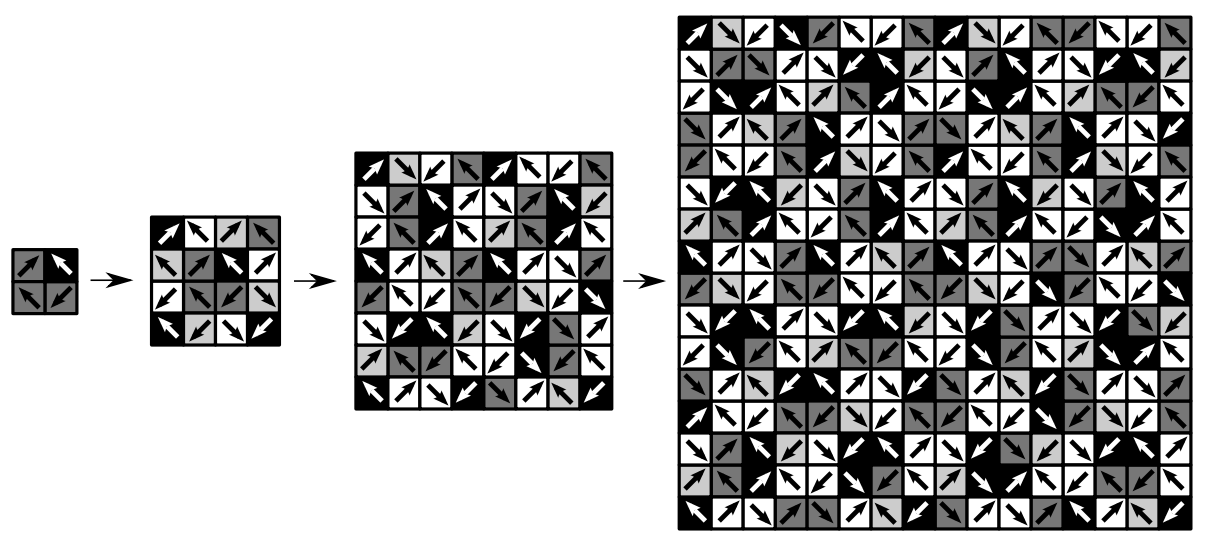

Figure 9. The iterates under $\sigma$ of the small patch on the left converge to $\mathcal{A}$.

Observe that $\sigma\left(R_{1}\right)$ contains $R_{2}^{\prime}$, and-more generally- $\sigma\left(R_{n}\right)$ contains $R_{n+1}^{\prime}$. (To see this one may use Figure 3 together with Figure 7.) Since $\sigma(\mathcal{S})=\mathcal{S}$ holds, the tiling $\mathcal{S}$ contains also the periodic arrangements from Equation (2). This shows that $\mathcal{S}$ and $\mathcal{A}$ coincide everywhere except on the diagonals $\{(x, y) \mid x= \pm y\}$. Considering the action of $\sigma$ it is easy to see that $\mathcal{S}$ has on each of the four branches of this set tiles with arrows that show in the same directions, and that these directions coincide with the ones in $\mathcal{A}$. Altogether we obtain $\mathcal{S}=\mathcal{A}$.

Note that there is also the concept of the hull $\mathbb{X}_{\sigma}$ of a substitution. Since $\mathcal{A}$ can be generated by a primitive substitution we have that $\mathbb{X}(\mathcal{A})=\mathbb{X}_{\sigma}$ (see [1] for details). More important, we now obtain the following result easily.

Theorem 3.4. All $\mathcal{T} \in \mathbb{X}(\mathcal{A})$ are aperiodic. In particular $\mathcal{A}$ is aperiodic (hence nonperiodic). 
Proof. Since $\sigma$ is a primitive substitution and $\mathbb{X}(\mathcal{A})$ is of finite local complexity, one can apply a classical result on substitution tilings [6, Thm 10.1.1]: a primitive substitution tiling is aperiodic if in this tiling the level 1 supertiles can be identified in a unique way. (See also Solomyak [16, Thm 1.1] for the proof of the "if and only if" version of this result.)

For the tilings in $\mathbb{X}(\mathcal{A})$ this is particularly simple: any level 1 supertile consists of four tiles sharing a common vertex. These four tiles have at least three distinct colours, and the arrows on the tiles do neither point to this common vertex nor do they point away from it. This leaves only one possibility: the vertices of the congruent copies of $R_{1}^{\prime}$ are the centres of the supertiles.

There are two geometric properties of a tiling $\mathcal{T}$ that have strong consequences on the dynamical properties of the hull $\mathbb{X}(\mathcal{T})$ of $\mathcal{T}$. A tiling $\mathcal{T}$ is repetitive if for each $r>0$ there is $R>0$ such that each patch of radius less than $r$ is contained in each patch of radius $R$. If $R \in O(r)$ then $\mathcal{T}$ is called linearly repetitive.

Moreover, a tiling $\mathcal{T}$ has uniform patch frequencies if the frequencies of all patches are well-defined. More precisely: if $P$ is a patch in $\mathcal{T}$ then let $N_{P}\left(B_{r}(x)\right)$ denote the number of congruent copies of $P$ in $\mathcal{T} \cap B_{r}(x)$, where $B_{r}(x)$ denotes the open ball of radius $r$ centred in $x$. If for all patches $P$ in $\mathcal{T}$ the limit

$$
\lim _{r \rightarrow \infty} \frac{1}{\pi r^{2}} N_{P}\left(B_{r}(x)\right)
$$

exists uniformly in $x$ then $\mathcal{T}$ has uniform patch frequencies. For a more thorough discussion of repetitivity or uniform patch frequencies see [1] or [4]. We omit it here since we need the terms only to state the following result.

Corollary 3.5. All $\mathcal{T} \in \mathbb{X}(\mathcal{A})$ are linearly repetitive and have uniform patch frequencies.

Proof. By a result of Solomyak [16, Lemma 2.3] each primitive substitution tiling in $\mathbb{R}^{2}$ with finite local complexity is linearly repetitive.

By a result of Lagarias and Pleasants [8, Theorem 6.1] linear repetitivity implies uniform patch frequencies.

The fact that our tilings possess the particular structure (union of periodic packings) described by Remark 3.2 is a hint that they are possibly limitperiodic. In this particular case this means that they can be generated by a certain cut-and-project method using a lattice in $\mathbb{R}^{2} \times\left(\mathbb{Q}_{2}\right)^{2}$, where $\mathbb{Q}_{2}$ denotes the field of 2-adic numbers. Structures of this kind are called model sets (with 2-adic internal space). Model sets are relevant since by a result of Hof [7, 14, 1] all models sets show pure point diffraction. It is beyond the scope of this paper to give details on this, but we may formulate the result and prove it using a simple to check sufficient condition.

Properly speaking, model sets are discrete point sets, not tilings. Hence our tilings are not model sets, but they are strongly related: they are mutually locally derivable (mld) with model sets, meaning that there is a local rule transforming one into the other (see [1]). 
Theorem 3.6. All $\mathcal{T} \in \mathbb{X}(\mathcal{A})$ are limitperiodic. Consequently, all $\mathcal{T} \in \mathbb{X}(\mathcal{A})$ are mutually locally derivable with model sets, hence pure point diffractive.

Proof. Since $\mathcal{A}$ is a primitive substitution tiling with integer scaling factor (in this case 2) and $\sigma$ is a block substitution (since each unit square is mapped to four unit squares in a $2 \times 2$ grid) we can apply a result in [9] and [10]: we need to show that $\sigma$ has a modular coincidence. By a result in [5] this is the case if there is a coincidence in the supertiles. For this it suffices to note that the upper right tile in $\sigma\left(T_{1}\right), \sigma\left(T_{2}\right), \sigma\left(T_{3}\right)$ and $\sigma\left(T_{4}\right)$ is a black tile $T_{1}$ with its arrow pointing down right, compare Figure 7 . (The lower left tile in all four cases is $T_{2}$, yielding a second coincidence.) For further details see [9, 10, 5, 1].

The fact that $\mathcal{A}$ is pure point diffractive has consequences if one imagines $\mathcal{A}$ as a physical solid: assume that in such a solid the four different types of atoms (or molecules) are arranged in the same pattern as the four different tiles in $\mathcal{A}$. A diffraction experiment would then show a diffraction image with bright spots ("Bragg peaks") and (ideally) no diffuse parts.

Now that we have obtained several results on the arrowed tiling $\mathcal{A}$ we turn our attention to the naked tiling using no decoration at all, compare Figure 2, Let us called the tiling obtained by the same construction but with undecorated squares $\mathcal{N}$.

A tiling $\mathcal{T}_{1}$ is called locally derivable from a tiling $\mathcal{T}_{2}$ if there is a local rule transforming $\mathcal{T}_{2}$ into $\mathcal{T}_{1}$. Two tilings $\mathcal{T}_{1}, \mathcal{T}_{2}$ are called mutually locally derivable if $\mathcal{T}_{1}$ is locally derivable from $\mathcal{T}_{2}$ and vice versa [1].

Lemma 3.7. The naked tiling $\mathcal{N}$ is locally derivable from the arrowed tiling $\mathcal{A}$.

Proof. It suffices to give a local rule to transform $\mathcal{A}$ into $\mathcal{N}$. Considering that in the arrow decoration of $\mathcal{A}$ the arrows point always away from the centre of the large square, the arrows determine locally the edges of the large (overlapping) squares in a unique way. The edges of the overlapping squares determine $\mathcal{N}$.

Two tilings that are mutually locally derivable share a lot of properties (aperiodicity, repetitivity, pure point diffraction, uniform patch frequency...) Thus it would suffice to give a local rule that transforms $\mathcal{N}$ into $\mathcal{A}$. We probably found such a rule, but unfortunately we were yet unable to prove that it really works.

Problem: Are $\mathcal{A}$ and $\mathcal{N}$ mutually locally derivable?

As an intermediate step, one may consider whether $\mathcal{A}$ is mutually locally derivable with the tiling obtained from $\mathcal{A}$ by deleting the colours. It is a simple exercise to see that this is indeed true.

\section{Remarks AND OutLOOK}

The original construction of inductive rotation tilings found by the second author used to place the origin close to the leftmost part of the patches $P_{n}$. Hence the "limit" of 
this sequence fills only a quarter plane. Four copies of this limit could be used to fill the entire plane. The resulting tilings are the same as the ones described in this paper. The construction in this paper is more adapted to the notion of convergence of sequences of tilings used here.

Here we showed that the tiling $\mathcal{A}$ is nonperiodic by showing that $\mathcal{A}$ is a substitution tiling and then applying the result of Solomyak that a substitution tiling is nonperiodic if $\sigma^{-1}$ is unique. We found an alternative proof of nonperiodicity by direct means, using only the limitperiodic structure of $\mathcal{A}$. For the sake of briefness we omit the alternative proof here.

The construction presented here uses squares and rotations by $\frac{\pi}{2}$. The second author found similar constructions for triangles and rotations by $\frac{2 \pi}{3}$ as well as for hexagons and rotations by $\frac{\pi}{3}$. For an artistic application of these constructions see http://hof stetterkurt.net/ip, see also [11.

The substitution rule $\sigma$ used to generate the tiling $\mathcal{A}$ uses four prototiles, but $\sigma\left(T_{3}\right)=\sigma\left(T_{4}\right)$. Probably one of these tiles is redundant and everything works for a substitution for three prototiles as well. We prefer to use the four-colour version since it carries more information, so it might make some arguments more clear.

We studied the tilings $\mathcal{A}$ and $\mathcal{N}$. We have good evidence (but no proof so far) that $\mathcal{A}$ and $\mathcal{N}$ are mutually locally derivable. What about other decorations? There are decorations of the large square $Q$ leading to 2-periodic tilings. So the construction yields at least two distinct mld classes (one aperiodic, one 2-periodic). Are there more mld classes?

The tilings $\mathcal{N}$ and $\mathcal{A}$ are obtained by looking on $R_{n}$ from "above". Are the tilings obtained by looking from below congruent to $\mathcal{N}$ respectively $\mathcal{A}$ ? Are they at least mutually locally derivable with $\mathcal{N}$ respectively $\mathcal{A}$ ?

We may realise the sets $R_{n}$ also with tiles with "thickness" in $\mathbb{R}^{3}$. Now above and below have a precise meaning. The construction for the sets $R_{n}$ now has to be described in three dimensions. Is there such a construction such that the height of all $R_{n}$ is bounded by a common constant? Are there arbitrary high stairs?

\section{ACKNOWLEDGEMENT}

The second author wants to express his gratitude to the many people from Mathematics, Art, Computer Science and Textile Technology who are supporting the work on Inductive Rotation Tilings, enabling new applications of aperiodic tilings in Arts and Science.

\section{REFERENCES}

[1] M. Baake, U. Grimm: Aperiodic Order. A Mathematical Invitation, Cambridge University Press (2013).

[2] N.P. Dolbilin, J.C. Lagarias, M. Senechal: Multiregular point systems, Discrete Comput. Geom. 20 (1998) 477-498.

[3] D. Frettlöh, F. Gähler, E. Harriss: Tilings Encyclopedia, available online:

http://tilings.math.uni-bielefeld.de 
[4] D. Frettlöh, C. Richard: Dynamical properties of almost repetitive Delone sets, Discr. Cont. Dynam. Syst. 33 (2014) 531-556.

[5] D. Frettlöh, B. Sing: Computing modular coincidences for substitution tilings and point sets, Discrete Comput. Geom. 37 (2007) 381-407.

[6] B. Grünbaum, G.C. Shephard: Tilings and Patterns, W.H. Freeman, New York (1987).

[7] A. Hof: On diffraction by aperiodic structures, Commun. Math. Phys. 169 (1995) 25-43.

[8] J.C. Lagarias and P.A.B. Pleasants: Repetitive Delone sets and quasicrystals, Ergodic Theory Dynam. Systems 23 (2003) 831-867.

[9] J.-Y. Lee, R.V. Moody, B. Solomyak: Pure point dynamical and diffraction spectra, Ann. Henri Poincaré 3 (2002) 1003-1018.

[10] J.-Y. Lee, R.V. Moody, B. Solomyak: Consequences of pure point diffraction spectra for multiset substitution systems, Discrete Comput. Geom. 29 (2003) 525-560.

[11] S. Parzer: Irrational Image Generator, Diploma thesis, Vienna University of Technology (2013).

[12] R. Penrose: The rôle of aesthetics in pure and applied mathematical research, Bull. Inst. Math. Appl. 10 (1974) 266-271.

[13] C. Radin, M. Wolff: Space tilings and local isomorphism, Geom. Dedicata 42 (1992) 355-360.

[14] M. Schlottmann: Generalised model sets and dynamical systems, in: M. Baake, R.V. Moody (eds.) Directions in Mathematical Quasicrystals, CRM Monograph Series, vol. 13, AMS, Providence RI (2000) pp. 143-159.

[15] D. Shechtman, I. Blech, D. Gratias, J.W. Cahn: Metallic phase with long-range orientational order and no translational symmetry, Phys. Rev. Lett. 53 (1984) 1951-1953.

[16] B. Solomyak: Non-periodicity implies unique composition property for self-similar translationally finite tilings, Discrete Comput. Geom. 20 (1998) 265-279.

[17] Wikipedia: Substitution Tiling, http://en.wikipedia.org/wiki/Substitution_tiling version of 16. Sept. 2014.

TeChnische FAKUltät, Universität Bielefeld, www.math.uni-bielefeld.de/ frettloe

WIEN, www. sunpendulum.at 